\title{
Transcription and Expression of Major VP Gene of Bombyx Mori Parvo-like Virus
}

\author{
Jinsong Cheng \& Qin Yao (Corresponding author) \\ Institute of Life Sciences, Jiangsu University \\ 301 Xuefu Road, Zhenjiang 212013, China \\ Tel: 86-511-8879-1923 E-mail: yaoqin@ujs.edu.cn \\ Meng Lv, Chen Sun, Yuanqing He \& Keping Chen \\ Institute of Life Sciences, Jiangsu University \\ 301 Xuefu Road, Zhenjiang 212013, China
}

The research is financed by the Program of the National Natural Science Foundation (30871826) and the National High Technology Research and Development Program of China (2008AA10Z145).

\begin{abstract}
The Bombyx mori parvo-like virus (China Zhenjiang isolate), termed as BmDNV-Z, replicates only in host's midgut columnar cells and causes flacherie disease. The viral genome is composed of two sets of different single-stranded linear DNA molecules, Viral DNA 1(VD1) and Viral DNA 2(VD2). Here we cloned the major VP gene from the plasmid contained viral DNA fragments. The gene consists of 1500 nucleotides and the deduced protein has 499 amino acid residues, with the predicted molecular weight of 54934.24Da, isoelectric point of 6.73. The coding sequence was inserted into expression plasmid vector pET-30a and expressed in Escherichia coli BL21 (DE3) under the inducible promoter LacZ. The expressed product was detected by Western blotting with anti-His antibody. Interestingly, there are four laddered band instead of one. We speculated that this virus may use leaky scanning mechanism to express four proteins in one ORF. And we investigated the major VP gene transcriptional time phase in susceptible silkworm through real time quantitative PCR (qPCR). The result showed that the mRNA level of VP gene increased greatly after 24 hours post inoculation.
\end{abstract}

Keywords: Bombyx mori parvo-like virus, Major VP protein gene, Transcription, Expression, qPCR

\section{Introduction}

Bombyx mori is not only an economic insect but also a model in life sciences. Bombyx mori parvo-like virus, termed as $B m \mathrm{DNV}-2$ (Yamanashi isolate) or BmDNV-Z (China Zhenjiang isolate), is one of the most disastrous viruses in cocoon production. Similar to the typical symptom in the host of silkworm infected by the BmDNV-1(Ina Isolate) (Bando H, 1987, 553-560), the BmDNV-2 also infected the columnar cells of midgut epithelium and caused flacherie disease of silkworm (Bando H, 1992 187-193). The most obvious characteristic in the genome of BmDNV-2 is that it has 2 sets of DNA molecular (VD1, VD2), and each of them is encapsidated respectively in the form of single-stranded liner DNA (+VD1,-VD1, +VD2,-VD2) so the BmDNV- 2 has 4 kinds of virions. Furthermore the sequence of BmDNV-2 is able to encode DNA polymerase itself (Bando, H., 1992 187-193; Bando, H. 1995 1147-11; Hayakawa Tohru et al 200066 101-108). BmDNV-2 has six structural proteins comprising four smaller structural proteins (VP1, 2, 3, and 4) with molecular weights around $50 \mathrm{kDa}$, and two larger ones with molecular weights around $110 \mathrm{kDa}$. The four smaller structural proteins are the major structural proteins, and amino acid sequencing and peptide mapping have revealed that VP1 to 4 are encoded in VD1. VP5 and 6 are rather minor components found in some purified virion fractions (Hayakawa Tohru, et al 200066 101-108). These unusual properties for a parvo-like virus imply an infection and replication mechanism different from parvovirus.

In 2005, the genome analysis of BmDNV-Z was completed by our laboratory (GenBank accession no. DQ017268 and 
DQ017269).Sequence analysis showed that VD1 genome consists of 6,543 nts and VD2 genome consists of 6,022 nts. Comparison of the complete genome sequence between BmDNV-Z and BmDNV-2 showed an identity of $98.4 \%$ in VD1 and $97.7 \%$ in VD2, with a total number of 228 bp substitutions, 11 bp deletions and $3 b p$ insertions found in BmDNV- Z (Wang et al., 2007, 35:103-108) The VP-ORF is located on the VD1 of BmDNV-Z ranging form $1417 \mathrm{nts}$ to $2916 \mathrm{nts}$. $B m \mathrm{DNV}-\mathrm{Z}$ has a high homology with $B m \mathrm{DNV}-2$. It implicated that $B m \mathrm{DNV}-\mathrm{Z}$ may share the same mechanism in gene expression and virion packaging with $B m \mathrm{DNV}-2$. Very little is known about the Transcription and Expression of Major VP Proteins of Bombyx mori Parvo-like Virus

In this paper, we investigated the major VP gene transcriptional time phase in susceptible silkworm through real time quantitative PCR (qPCR). The major VP gene of BmDNV-Z was expressed in Escherichia coli BL21 (DE3) and the fusion protein was detected by Western blotting with anti-His antibody. The result showed that the mRNA level of VP gene increased greatly after 24 hours post inoculation. The expression of recombinant VP tagged with 6 histidines was confirmed by Western blotting and there were four clearly distinct polypeptide bands. In light of above, we infer that this virus may use leaky scanning mechanism to express four proteins in one ORF.

\section{Materials and methods}

\subsection{Cloning of VP gene and Construction of expression plasmid}

ORF region of the major VP gene of $B m$ DNV-Z was amplified by PCR using the forward primer

\section{5 'GCGGATCCATGGGTAGAGTACTTGGCTCATT3` and the reverse primer 5 'GCAAGCTTTTTAT}

GAAAACCAACAAGCCT3` containing BamHI and HindIII restriction sites (underlined) respectively. PCR reaction was carried out for 35 amplification cycles $\left(94^{\circ} \mathrm{C} / 30 \mathrm{sec}, 58^{\circ} \mathrm{C} / 30 \mathrm{sec}, 72^{\circ} \mathrm{C} / 1.5 \mathrm{~min}\right)$. The PCR product was ligated into pMD19-T (TaKaRa, Dalian) vector using T4 DNA ligase (TaKaRa, Dalian) and then transformed into E. coli (TG1 strain). Sequencing was performed in Shanghai Sangon Bio-technology Corporation using the plasmid pMD19-vp.

\subsection{Expression of VP fusion protein in E. coli}

After digestion with BamH I and Hind III from the plasmid pMD19-vp, the purified DNA fragment was ligated into expression vector pET-30a (Novagen) and introduced into E.coli BL21 (DE3) by transformation. The vector pET-30a can express fusion protein with both the N-terminal and C-terminal His-tag. We deleted the stop codon of the CDS of the VP and allowed the fusion protein has both $\mathrm{N}$-terminal and $\mathrm{C}$-terminal His-tag. So the fusion protein can be purified through His purification system. The expression of recombinant VP was induced overnight at $28^{\circ} \mathrm{C}$ by the addition of isopropyl- $\beta$-D-thiogalactopyranoside (IPTG) to a final concentration of $1.0 \mathrm{mM}$ when the optical density of the culture at $600 \mathrm{~nm}$ reached 0.5 . A $10 \%$ sodium dodecyl sulfate-polyacrylamide gel electrophoresis (SDS-PAGE) was performed to analyze the fusion protein.

\subsection{Western blot analysis}

To identify the fusion protein, the western blotting was performed using anti-His-tag antibody (TIANGEN, Beijing) following the standard protocol.

\subsection{Transcriptional time phase of VP gene by $q P C R$}

The total RNA was extracted from infected midgut tissue of susceptible silkworm strain HUABA35 at 12 time points (2, $4,6,12,24,28,36,40,48,52,66,72 \mathrm{~h}$ post inoculation) using a Trizol reagent (Invitrogen, Carlsbad, CA), according to the manufacturer protocol. The total RNA subjected to messenger polyA RNA isolation using the Oligo Tex mRNA Mini Kit (QIAGEN Inc. Valencia, CA). Finally, the RNA was dried and resuspended in DEPC- $\mathrm{H}_{2} \mathrm{O}$ containing $1 \%$ RNase inhibitor and stored at $-80^{\circ} \mathrm{C}$. The first-strand cDNA synthesis was performed SYBR RT-PCR Kit A (TaKaRa) at $37^{\circ} \mathrm{C}$ for $1 \mathrm{~h}$ in a final volume of $20 \mu \mathrm{l}$ reaction buffer

QPCR was conducted on an MX3000P thermal cycler amplification and detection system (Stratagene). QPCR amplification of the cDNA was performed using the SYBR RT-PCR Kit B (TaKaRa). Specific primers for virus gene vp is: the forward primer 5'GGTACGATGTTGACCCAG3' and the reverse primer 5'ACTCCACCAGCAAAGAC3', Each reaction was performed in a $20 \mu \mathrm{l}$ reaction volume which contained $10 \mu \mathrm{l}$ of SYBR Premix Ex Taq; $1 \mu \mathrm{M}$ of each upstream and downstream primer and $1 \mu \mathrm{l}$ cDNA templates diluted first-strand cDNA (1/10). Preincubation was performed for $30 \mathrm{~s}$ at $95^{\circ} \mathrm{C}$ to denature the template DNA and to activate Taq-polymerase. DNA was amplified for 40 cycles of $10 \mathrm{~s}$ at $95^{\circ} \mathrm{C}, 20 \mathrm{~s}$ at $56^{\circ} \mathrm{C}$, and $20 \mathrm{~s}$ at $72^{\circ} \mathrm{C}$.

The primers' design was based on the complete genome sequence of BmDNV-Z (GenBank accession no. DQ017268). Primers were designed by using the software Primer Premier 5. Two pairs of primers were located on the ORF3 region of the BmDNV-Z VD1 genomic sequence. BmactinA3 gene was used as an internal control for normalization. Actin A3 was amplified using the forward primer 5'GCGCGGCTACTCGTTCACTACC3'and the reverse primer 5'GGATGTCCACGTCGCACTTCA3'. The specificity of the primers was confirmed by using NCBI BLAST (BLASTN) algorithms. 


\section{Results and Discussion speculation}

The transcriptional time phase analysis of VP gene by qPCR indicated that transcription of VP gene start at 24 hours after inoculation in midgut tissue of susceptible silkworm (figure 5). After 52h the transcriptional level of VP gene increased greatly. This profile is consistent with the most of viral gene expression process. As a late gene the transcriptional level of VP gene is fairly low at the beginning, because the virus needs to save energy for perform viral DNA replication. After $52 \mathrm{~h}$ pi the virus prepares for packaging.

The limited complexity of parvovirus genome means that the virus must put its genes into full play. This strategy is implemented and orchestrated by a few multifunction viral regulatory protein (FeÂ dieÁ re, 2004, 181- 189) or adapts some kind of mechanism to generate more protein through less content of genome. The strategy of VP gene expression for most parvovirus including Densovirus (e.g. Bombyx mori Densovirus 1, Casphalia extranea Densovirus, Galleria mellonella Densovirus) is leaky scanning mechanism, which was confirmed through western blotting. (Sotoshiro H, 1995,66 :60 - 67, Hayakawa T, et al, 2000, 101-108. G. FeÂ dieÁ re, \& Y. Li 2002, 299-308; P. Tijsse \& Y. Li, 2003, 10357-10365). By the Leaky scanning model the virus can generate about four structural proteins from a singe transcript. Each additional peptide extension is thought to fulfill at least one unique function in the viral cycle. These strategies also allow the virus to express the different domains to different optimal amounts. The reason why the virus uses leaky scanning is that the virus must put its genes into full play with one mRNA to produce one wholesome protein and three N-terminal truncated proteins. The expression level suggested a 2-5 fold increase in leaky scanning (Byung-Sik Shin, 2002, 1015-1025).

In this experiment the viral VP protein was expressed in prokaryotic and analyzed by SDS-PAGE and detected by using Western blotting (figure 4). Four clearly distinct polypeptide bands were witnessed with protein molecular mass approximately $64 \mathrm{kDa}(\mathrm{VP} 1), 55 \mathrm{kDa}(\mathrm{VP} 2), 41 \mathrm{kDa}(\mathrm{VP} 3), 34 \mathrm{kDa}$ (VP4) respectively. The open reading frame starts at nucleotide 1 (1AUG), 115(115AUG), 486(486AUG), 657(657AUG) on the ORF sequence of VP gene. Predictions of the molecular masses of the four structural proteins from the first four in-frame AUGs in the VP ORF corresponded well with the values seen in SDS-PAGE analysis. These results implied that the Bombyx mori parvo-like virus (China Zhenjiang isolate) may use leaky scanning mechanism to generate four N-terminal truncated VP proteins. As the vector pET-30a can express fusion protein with both the N-terminal and C-terminal His-tag. We reserved the C-terminal His-tag sequence through deleting the stop codon of the CDS of the VP. In this way, the fusion protein VP1 has both $\mathrm{N}$-terminal and C-terminal His-tag, and the VP2, VP3 and VP4 have C-terminal His-tag. So all of them can be detected by Western blotting with His-tag antibody.

The results also showed that the VP1 and VP3 are the main components of the viral structural protein and the VP2 and VP4 are much less than VP1 and VP3 in amount. This phenomenon indicated that the initiation codons of VP1 and VP3 were preferentially expressed. The genome of plum pox virus contains a single open reading frame that is translated into a large polyprotein. Although the open reading frame starts at nucleotide 36 (36AUG), it is translated from the second, 147AUG, which is in a more favorable context for translation initiation. When the cryptic 36AUG was placed in a favorable context, it turned into an efficient initiation codon in vitro. Furthermore, AUGs that were placed in a favorable context, initiating short intraleader open reading frames, repressed translation initiation from the 147AUG in vitro and in vivo. These results pointed to leaky scanning as the mechanism of translation initiation of plum pox virus RNA. Nevertheless, it is a peculiar leaky scanning where the initiation of translation does not require a cap structure at the 5 ' end. (Simón-Buela L, Guo HS, García JA.1997 2691-9.). Western blotting revealed that translation of these VP transcripts of Casphalia extranea Densovirus was not restricted to the first AUG but that it also led to recognition of downstream initiation codons to yield all structural proteins contained in the expressed transcript. Initiation codons 3

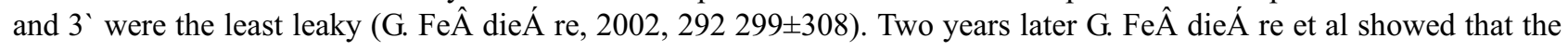
densonucleosis virus of Mythimna loreyi use the same expression strategy to generate VP3 and VP4 yielded background products that were smaller than VP4 but reacted with the virus-specific antiserum. It appeared that the VP4 codon became somewhat leaky in the absence of the VP1 and VP2 initiate codon. (G. FeÂ dieÁ re, 2004, 181- 189) by bioinformatics analysis. The short untranslated 5 leader sequence and the less favorable contexts of VP2 and VP4 AUG initiation codons were found in VP gene of $B m \mathrm{DNV}-\mathrm{Z}$

It is the first time that the leaky scanning mechanism of major VP gene of $B m \mathrm{DNV}-\mathrm{Z}$ was witnessed by western blotting. Nevertheless, it would be possible that the set of VPs is generated by proteolysis of the largest VP (VP1), translation downstream of (an) internal ribosome entry site(s), or ribosomal shunting. As an economic and model insect of Lepidoptera, research for B.mori and related genes has attracted more and more attention. Specially, the VP gene may have some unknown functions. The transcription and expression analysis can help us to do further study about this gene. The details of capsid assembly mechanism and the interactions between VP and silkworm cell protein is in progress.

\section{References}

Bando H, Kusuda J, Gojobori T, Maruyama T, \& Kawase S. (1987). Organization and nucleotide sequence of a densovirus genome imply a host-dependent evolution of the parvoviruses. Journal of virology Feb; 61(2):553-560. 
Bando, H., Choi H., Ito, Y., Nakagaki, M., \& Kawase, S. (1992). Structural analysis on the single-stranded genomic DNAs of the virus newly isolated from silkworm: the DNA molecules share a common terminal sequence. Arch. Virol. 124: $187-193$.

Bando, H., Hayakawa, T., Asano, S., Sahara, K., Nakagaki, M., \& Iizuka, T. (1995). Analysis of the genetic information of a DNA segment of a new virus from silkworm. Arch. Virol. 140: 1147-11

Byung-Sik Shin, David Maag, Antonina Roll-Mecak, M. Shamsul Arefin, Stephen K. Burley, Jon R. Lorsch, \& Thomas E. Dever. (2002). Uncoupling of Initiation Factor eIF5B/IF2 GTPase and Translational Activities by Mutations that Lower Ribosome Affinity Cell, Volume 111, Issue 7, 27 December, Pages 1015-1025

G. FeÂ dieÁ re, M. El-Far, Y. Li, M. Bergoin, \& P. Tijssen. (2004). Expression strategy of densonucleosis virus from Mythimna loreyi Virology 320 181- 189

G. FeÂ dieÁ re, Y. Li, Z. ZaÂ dori, J. Szelei, \& P. Tijssen. (2002). Genome Organization of Casphalia extranea Densovirus, a New Iteravirus Virology 292, 299-308.

Li Y, Zadori Z, Bando H, R. Dubuc, G. FeÂ dieÁ re, J. Szelei \& P. Tijssen. (2001). Genome organization of the densovirus from Bombyx mori (BmDNV-1) and enzyme activity of its capsid. The Journal of general virology Nov; 82 (Pt 11):2821-2825.

Hayakawa T, Kojima K, \& Nonaka K, et al. (2000). Analysis of proteins encoded in the bipartite genome of a new type of parvo-like virus isolated from silkworm structural protein with DNA polymerase motif. Virus Res Jan,, 66 (1):101-108.

P. Tijssen, Y. Li, M. El-Far, J. Szelei, M. Letarte, \& Z. Za'dori. (2003). Organization and Expression Strategy of the Ambisense Genome of Densonucleosis Virus of Galleria mellonella JOURNAL OF VIROLOGY, 19, $10357-10365$.

Simón-Buela L, Guo HS, \& García JA. (1997). Cap-independent leaky scanning as the mechanism of translation initiation of a plant viral genomic RNA. J Gen Virol. Oct;78 (Pt 10):2691-9.

Sotoshiro H, Kobayashi M1. (1995). Identification of viral structural polypeptides in the midgut and feces of the silkworm Bombyx mori, infected with Bombyx Densovirus Type 2 J Invertebr Pathol, 66 :60 - 671

Wang, Yongjie, Yao, Qin, Chen, Keping, Wang, Yong, Lu, Jian, \& Han, Xu. (2007). Characterization of the genome structure of Bombyx mori densovirus (China isolate) Virus Genes 35:103-108 DOI 10.1007/s11262-006-0034-3

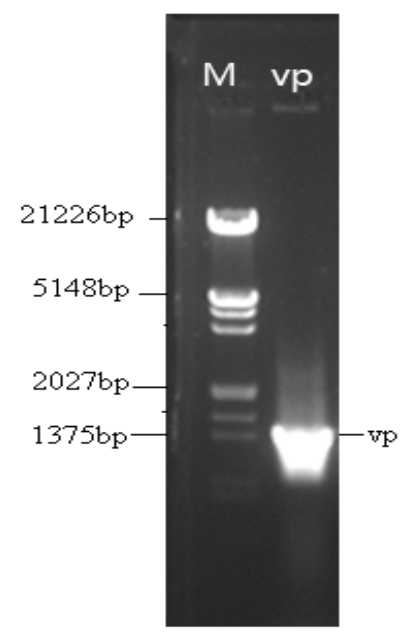

Figure 1. The result of PCR for vp. Lane M, DNA molecular mass maker; Lane vp, PCR product of vp (1500bp). 


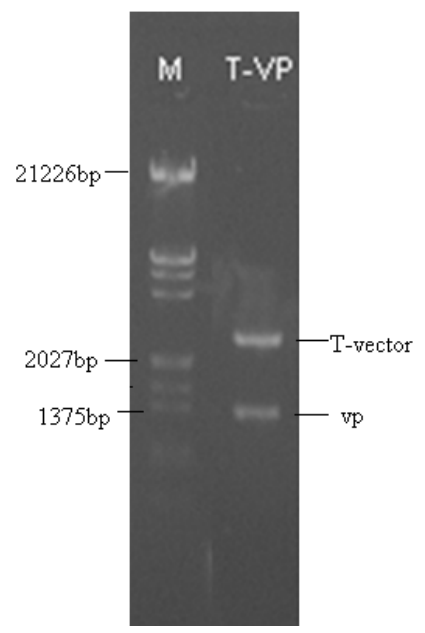

Figure 2. Identification of the recombinant plasmid pMD19-T/vp. Lane M, DNA molecular mass maker. Lane T- $v p$, pMD19-T/ $v p$ digested with BamHI and HindIII generated two fragments: pMD19-T (2.6 Kbp) and vp (1500 bp);

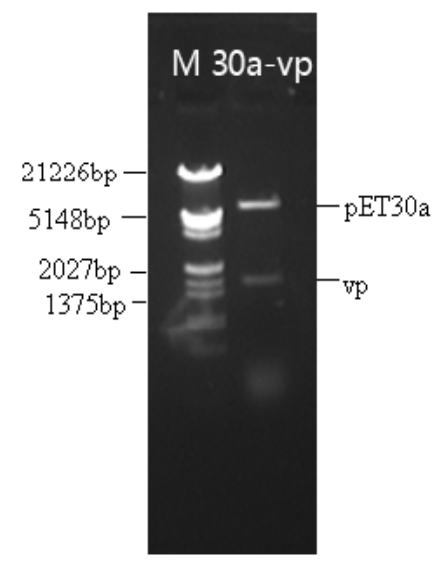

Figure 3. Identification of the expression vector His- $v p$-pET30a. Lane M, DNA molecular maker. Lane 30a-vp, His- $v p$-pET30a digested with BamHI and hindIII generated two fragments: His-pET30a (5.4Kbp) and vp (1500 bp).

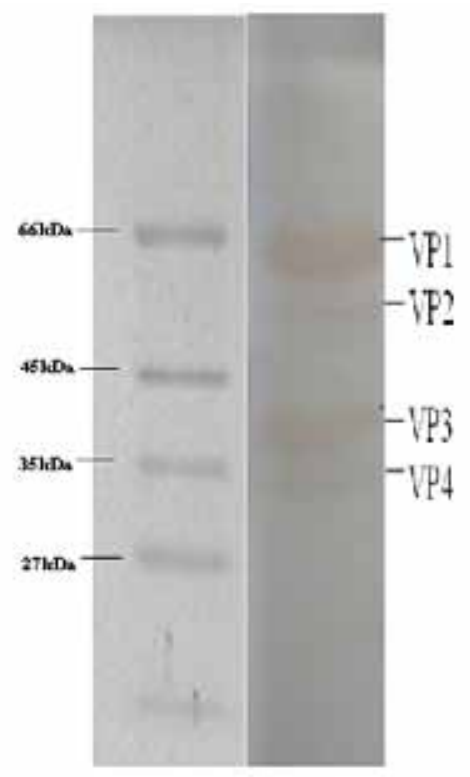

Figure 4. Western blotting analysis. Protein of E. coli BL21 contained His-pET30a induced by IPTG; Protein of E. coli BL21 contained His-GlcAT-S-pET30a induced by IPTG Western blot results of His-VP fusion protein. The four fusion protein bands were indicated by arrows. 


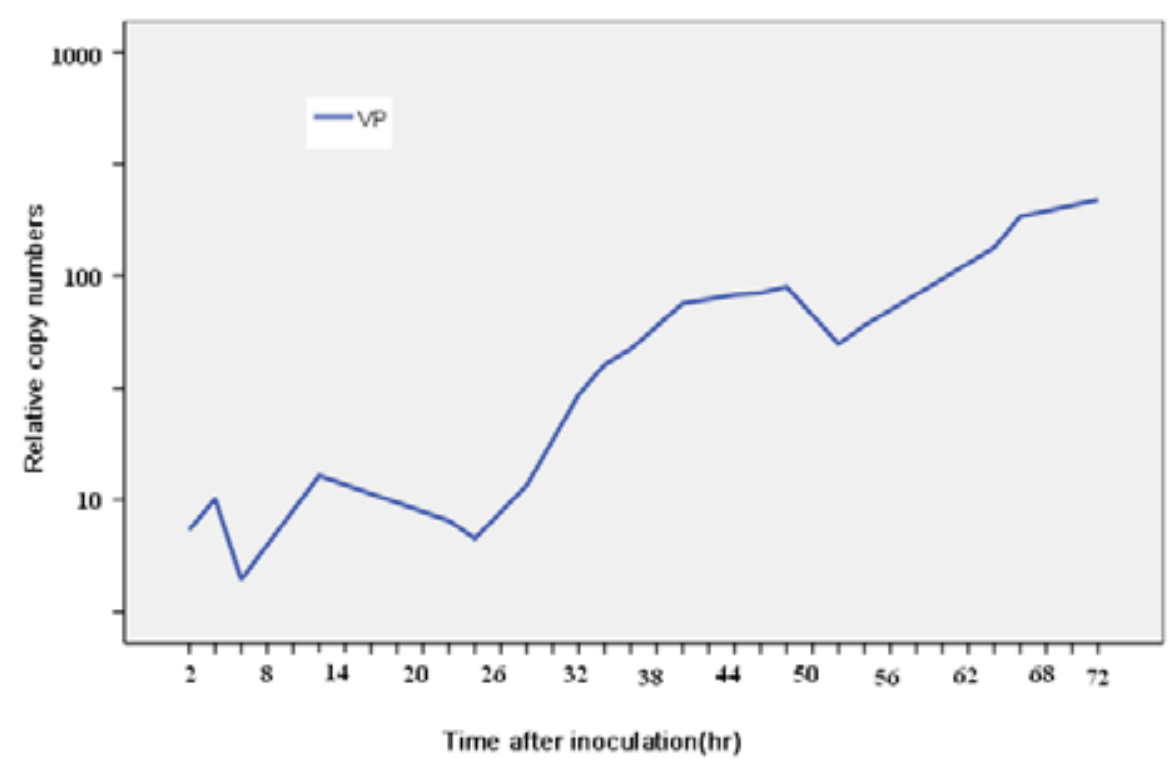

Figure 5. The mRNA level of BmDNV-Z VP protein. The copy numbers of vp mRNA increase considerably after $24 \mathrm{hrs}$. $B m a c t i n A 3$ gene was used as an internal control for normalization. 